\title{
Sources of electron pitch angle anisotropy in the magnetotail plasma sheet
}

\author{
Andrew P. Walsh, ${ }^{1}$ A. N. Fazakerley, ${ }^{2}$ C. Forsyth, ${ }^{2}$ C. J. Owen, ${ }^{2}$ M. G. G. T. Taylor, ${ }^{1}$ \\ and I. J. Rae ${ }^{2}$ \\ Received 27 June 2013; revised 6 September 2013; accepted 6 September 2013; published 1 October 2013.
}

[1] We survey the properties of electron pitch angle distributions in the magnetotail plasma sheet at a distance between 15 and $19 R_{E}$ from the Earth, using data from the Plasma Electron and Current Experiment (PEACE) instrument. We limit our survey to those pitch angle distributions measured when the interplanetary magnetic field (IMF) had been steadily northward or steadily southward for the previous $3 \mathrm{~h}$. We find that, at sub-keV energies, the plasma sheet electron pitch angle distribution has an anisotropy such that there is a higher differential energy flux of electrons in the (anti-) field-aligned directions. Fitting the measured pitch angle distributions with both a single and two component kappa distribution reveals that this anisotropy is the result of the presence of a second, cold, component of electrons that is observed more often than not, and occurs during both the northward and southward IMF intervals. We present evidence that suggests the cold electron component has an ionospheric, rather than magnetosheath, source and is linked to the large-scale field-aligned current systems that couple the magnetosphere and ionosphere.

Citation: Walsh, A. P., A. N. Fazakerley, C. Forsyth, C. J. Owen, M. G. G. T. Taylor, and I. J. Rae (2013), Sources of electron pitch angle anisotropy in the magnetotail plasma sheet, J. Geophys. Res. Space Physics, 118, 6042-6054, doi:10.1002/jgra.50553.

\section{Introduction}

[2] The magnetotail plasma sheet is a region of enhanced plasma pressure in the magnetotail that exists on closed magnetic field lines on the nightside of the Earth's magnetosphere. The plasma sheet is separated from the magnetotail lobes by the plasma sheet boundary layer. The plasma sheet and plasma sheet boundary layer are typically thought to contain particle populations with different characteristics that themselves change with distance from the Earth [e.g., Wang et al., 2013]. At $\sim 20 R_{E}$ from the Earth, a region of the magnetotail comparatively well-sampled by spacecraft, the plasma sheet boundary layer is usually considered to be characterized by field-aligned or bidirectional particle distributions while the central plasma sheet has been more often observed to contain isotropic particle distributions [Eastman et al., 1984; Parks et al., 1984]. The evolution between fieldaligned and isotropic particles is thought to occur through nonadiabatic pitch angle scattering as the particles traverse the magnetotail current sheet [Tsyganenko, 1982; Sergeev et al., 1983; Walsh et al., 2011].

\footnotetext{
${ }^{1}$ Science and Robotic Exploration Directorate, European Space Agency, ESTEC, Noordwijk, The Netherlands.

${ }^{2}$ Department of Space and Climate Physics, Mullard Space Science Laboratory, University College London, Surrey, UK.

Corresponding author: A. P. Walsh, Science and Robotic Exploration Directorate, European Space Agency, ESTEC, Keplerlaan 1, 2201AZ Noordwijk ZH, Netherlands. (awalsh@rssd.esa.int)

(C)2013. American Geophysical Union. All Rights Reserved. 2169-9380/13/10.1002/jgra.50553
}

[3] Omnidirectional particle distributions in the plasma sheet have been well studied and are known to be suprathermal in character; they are well-described by kappa distributions with either a single or several components [e.g., Christon et al., 1989, 1991; Wang et al., 2007; Haaland et al., 2010]. However, there have been few statistical investigations of the properties of particle pitch angle distributions in the plasma sheet that extend beyond case studies. Wang et al. [2013] recently investigated how proton pitch angle anisotropy varied with distance from the Earth and geomagnetic activity, finding that ions with energies from $\sim$ $1 \mathrm{keV}$ up to $\sim 10 \mathrm{keV}$ were isotropic in the magneotail plasma sheet, consistent with the results of Walsh et al. [2011], who surveyed proton and electron pitch angle distributions (PADs) measured by the Cluster Ion Spectroscopy (CIS) and Plasma Electron and Current Experiment (PEACE) instruments. They found that, on average, the proton plasma beta, $\beta_{p}$, organizes the protons into the canonical plasma sheet boundary layer-central plasma sheet structure described above: Field-aligned distributions evolving into isotropic protons with increasing $\beta_{p}$. However, the same is not true of the electrons.

[4] Figure 3 of Walsh et al. [2011] is reproduced here in Figure 1. The average electron differential energy flux ( $\mathrm{keV} \mathrm{cm} \mathrm{cm}^{-2} \mathrm{~s}^{-1} \mathrm{sr}^{-1} \mathrm{keV}^{-1}$, dEF) is plotted as a function of $\beta_{p}$ and energy for electrons flowing earthward parallel or antiparallel to the magnetic field, depending on hemisphere (henceforth earthward; Figure 1a), perpendicular to the magnetic field (Figure 1b), and tailward parallel or antiparallel to the magnetic field, depending on hemisphere 


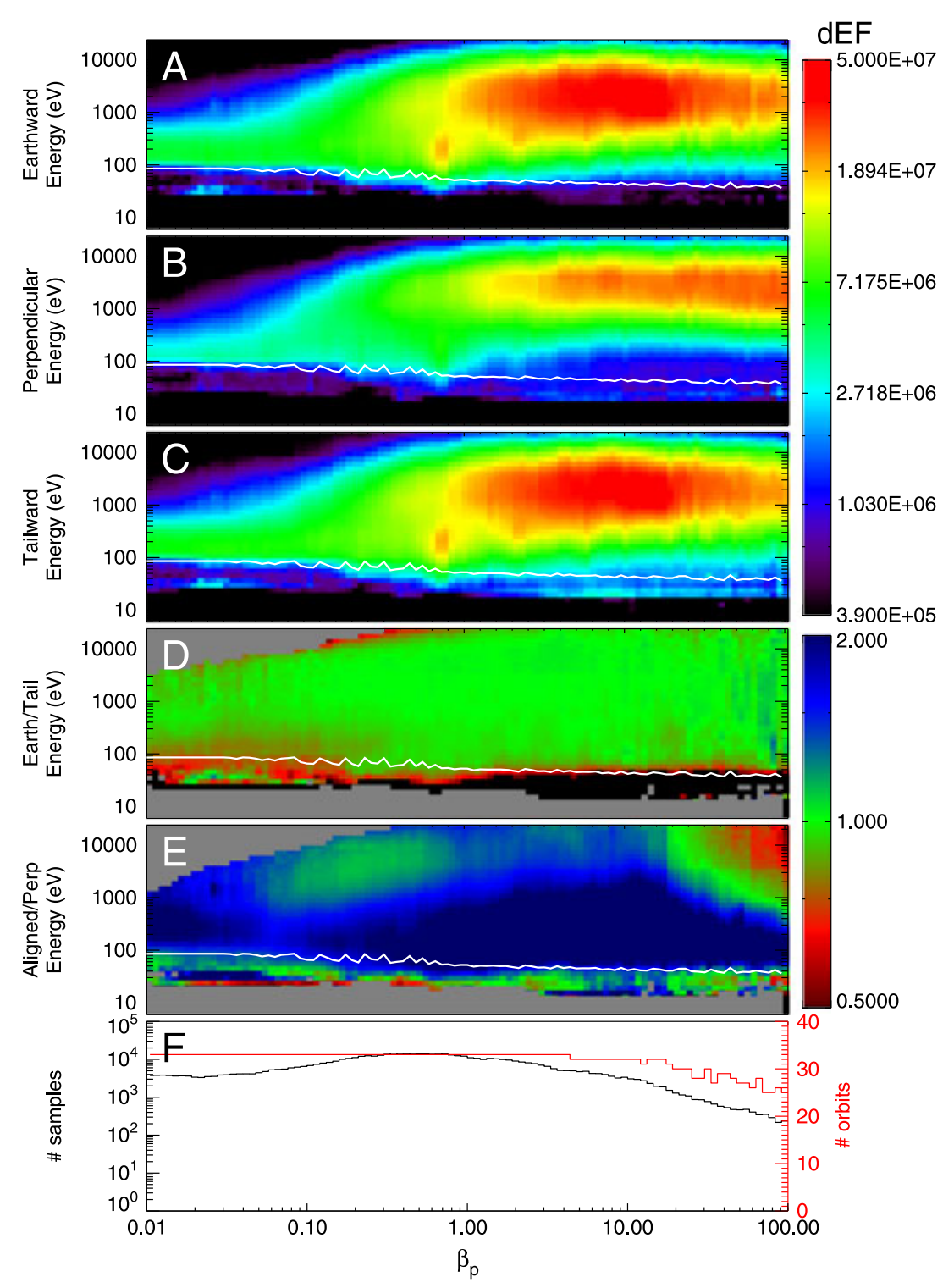

Figure 1. A reproduction of Figure 3 from Walsh et al. [2011]. Average electron differential energy flux (dEF) in the (a) earthward field-aligned, (b) perpendicular, and (c) tailward field-aligned directions are plotted as a function of proton plasma beta $\left(\beta_{p}\right)$ and energy. (d) The ratio between earthward and tailward dEF is plotted and (e) the ratio between field-aligned and perpendicular dEF is plotted. White lines overplotted represent the maximum spacecraft potential observed for that value of $\beta_{p}$.

(henceforth tailward; Figure 1c). The maximum spacecraft potential observed in a given $\beta_{p}$ bin is overplotted in white. The ratio between the earthward and tailward $\mathrm{dEF}$ is plotted in Figure $1 \mathrm{~d}$ and the ratio between the average field-aligned $\mathrm{dEF}$, i.e., (earthward + tailward) $/ 2$, and perpendicular $\mathrm{dEF}$ is plotted in Figure 1e. Isotropic electron PADs would have equal $\mathrm{dEF}$ in the field-aligned and perpendicular directions, and hence would appear green in Figure 1e. There are no isotropic electron PADs evident in Figure 1, even for values of $\beta_{p} \geq 3$, a range within which the protons have isotropized [Walsh et al., 2011]. Instead, the electron PADs in the magnetotail are, on average, dominated by fieldaligned, bidirectional electrons (green in Figure 1d, blue in Figure 1e). This is true for all but the highest values of $\beta_{p}$ $\left(\beta_{p} \geq 17\right)$, where instead the electron PADs are dominated by perpendicular electrons, signified by red in Figure 1e. Walsh et al. [2011] explained this difference in isotropy of protons and electrons in the magnetotail plasma sheet in terms of the increased likelihood for protons to behave nonadiabatically when compared to electrons [Tsyganenko, 1982; Sergeev et al., 1983] and hence are subject to pitch angle scattering in the magnetotail current sheet.

[5] The field-aligned anisotropy in plasma sheet electrons is most pronounced at sub-keV energies (Figure 1e), and it is known that the plasma sheet is composed of lower energy particles during undisturbed intervals [Christon et al., 1989] and northward interplanetary magnetic field (IMF) [e.g., Øieroset et al., 2005; Nishino et al., 2007a], so the apparent anisotropy found by Walsh et al. [2011] could simply be the result of the average PADs convolving 
a colder, field-aligned, electron plasma sheet population observed during quieter intervals and a hotter, more isotropic, electron plasma sheet population observed during disturbed intervals.

[6] Here we investigate this possibility and seek to identify the source of the anisotropy in the electron plasma sheet, by extending the Walsh et al. [2011] survey to include data from the 2001-2006 tail seasons, but limited to those intervals when the magnetosphere is exposed to either steady northward IMF or steady southward IMF. We find that, contrary to the above suggestion, the field-aligned anisotropy is the result of a cold component of electrons, most likely with its source in the ionosphere, that is present during both steady northward and steady southward IMF intervals.

\section{Data Selection and Processing}

[7] In order to study the behavior of plasma sheet electron PADs under different IMF conditions, we expand the data set used by Walsh et al. [2011] to include the 2001-2006 Cluster tail seasons (i.e., day of year 200-300 during each year). We employ magnetic field data from FGM (FluxGate Magnetometer) [Balogh et al., 2001], spacecraft potential data from EFW (Electric Fields and Waves) [Gustafsson et al., 2001], electron PADs from PEACE (Plasma Electron and Current Experiment) [Johnstone et al., 1997], and proton moments from CIS (Cluster Ion Spectroscopy) [Rème et al., 2001]. CIS data are from the CODIF (Composition and Distribution Function Analyser) sensor. All CIS moments were calculated on the ground from 3-D distributions. All data are from the Cluster 4 spacecraft. Cluster 4 was chosen over the other Cluster spacecraft because the CODIF sensor on that spacecraft has produced the most reliable data over the 6 year interval under investigation, and it has the most appropriate energy range for study of the plasma sheet. Data from only one spacecraft were used to avoid double counting PADs and to minimize errors introduced by imperfect cross-calibration of instruments. The years 2001-2006 were chosen as subsequently to this Cluster's orbit began to evolve such that its passage through the plasma sheet occurred much closer to Earth. IMF data are from NASA/Goddard Space Flight Center (GSFC)'s 1 min resolution OMNI data set.

[8] We limit our survey to those pitch angle distributions measured when the GSM $Z$ component of the IMF has been steadily positive (steady northward IMF) or negative (steady southward IMF) prior to each pitch angle distribution being measured. We note that the definition of steady northward or southward IMF can have a large effect on the results of a survey such as this. If the length of time that constitutes steady is chosen to be too long the resulting data set can be too small to produce significant results; conversely, if it is too short, even with a large data set, features associated with steady IMF may not have time to develop. Similarly, an overly restrictive IMF direction criterion can reduce the available data, while a more vague criterion can introduce noise. Here we define steady northward IMF as intervals where the mean of the IMF over the previous $3 \mathrm{~h}$, minus its standard deviation, is positive (i.e., $\left\langle B_{Z}\right\rangle_{3 H r}-\sigma_{B Z 3 H r}>0$ ). Equivalently, steadily southward IMF is defined as $\left\langle B_{Z}\right\rangle_{3 H r}+\sigma_{B Z 3 H r}<0$. The inclusion of the standard deviation term in the stability criterion has the effect of discounting those intervals where IMF $B_{Z}$ oscillated about zero, but had a small net positive or

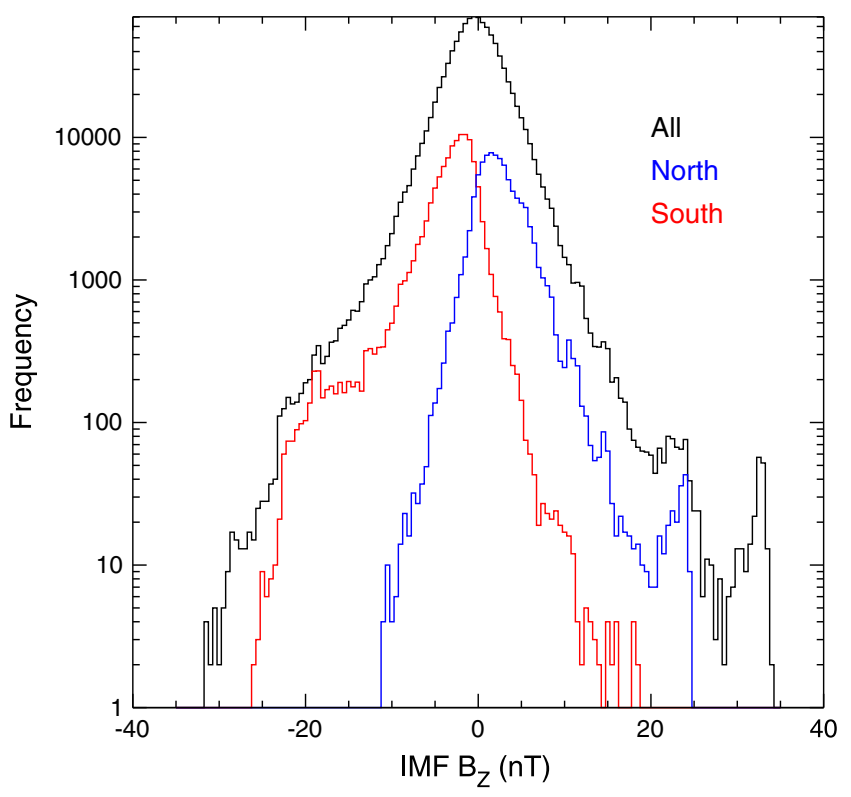

Figure 2. Histogram of IMF $B_{Z}$ values (GSM coordinates) at $1 \mathrm{~min}$ resolution for the 2001-2006 Cluster tail seasons (black), and for the steady northward (blue) and steady southward (red) intervals defined above. The bin size is $0.5 \mathrm{nT}$ in all cases.

negative bias. Three hours was chosen as a steady interval because at quiet-time convection speeds of $50 \mathrm{~km} \mathrm{~s}^{-1}$ [e.g., Baumjohann, 1993], a flux tube can travel $85 R_{E}$ in $3 \mathrm{~h}$, i.e., from near the distant neutral line [e.g., Nishida, 2000] to a position close to, or earthward of, Cluster apogee in the tail. The $\left\langle B_{Z}\right\rangle \pm \sigma_{B Z}$ criterion was chosen after some experimentation to provide a balance between a completely northward or southward IMF data set and allowing a data set large enough to produce meaningful results. Figure 2 shows the distribution of IMF $B_{Z}$ values in GSM coordinates for the six Cluster tail seasons considered in the survey (black), the steady northward (blue), and steady southward (red) subsets, as defined above. Both the steady northward and steady southward subsets contain $\sim 10 \%$ of the total interval, providing a data set roughly half the size of that used by Walsh et al. [2011] for each case. As can be seen from Figure 2, the steady northward (southward) subset does contain some southward (northward) IMF measurements. However, these make up only $12 \%(11 \%)$ of the selected data. This means that, on average, in the $3 \mathrm{~h}$ prior to the measurement of each of our selected pitch angle distributions, IMF $B_{Z}$ has been pointing the wrong way for only $20 \mathrm{~min}$ that need not be consecutive.

[9] The individual times (at 1 min resolution) prior to which the IMF was steadily northward or southward for $3 \mathrm{~h}$ were identified and sorted such that consecutive times were grouped together. In this way, 492 steady northward IMF intervals and 547 steady southward IMF intervals were identified. The intervals range from $1 \mathrm{~min}$ to $40 \mathrm{~h}$ in duration, with a median duration of $2 \mathrm{~h}$. For each interval, electron pitch angle distributions, proton pitch angle distributions, proton moments, the magnetic field vector, and the spacecraft potential were interpolated onto a common time grid 

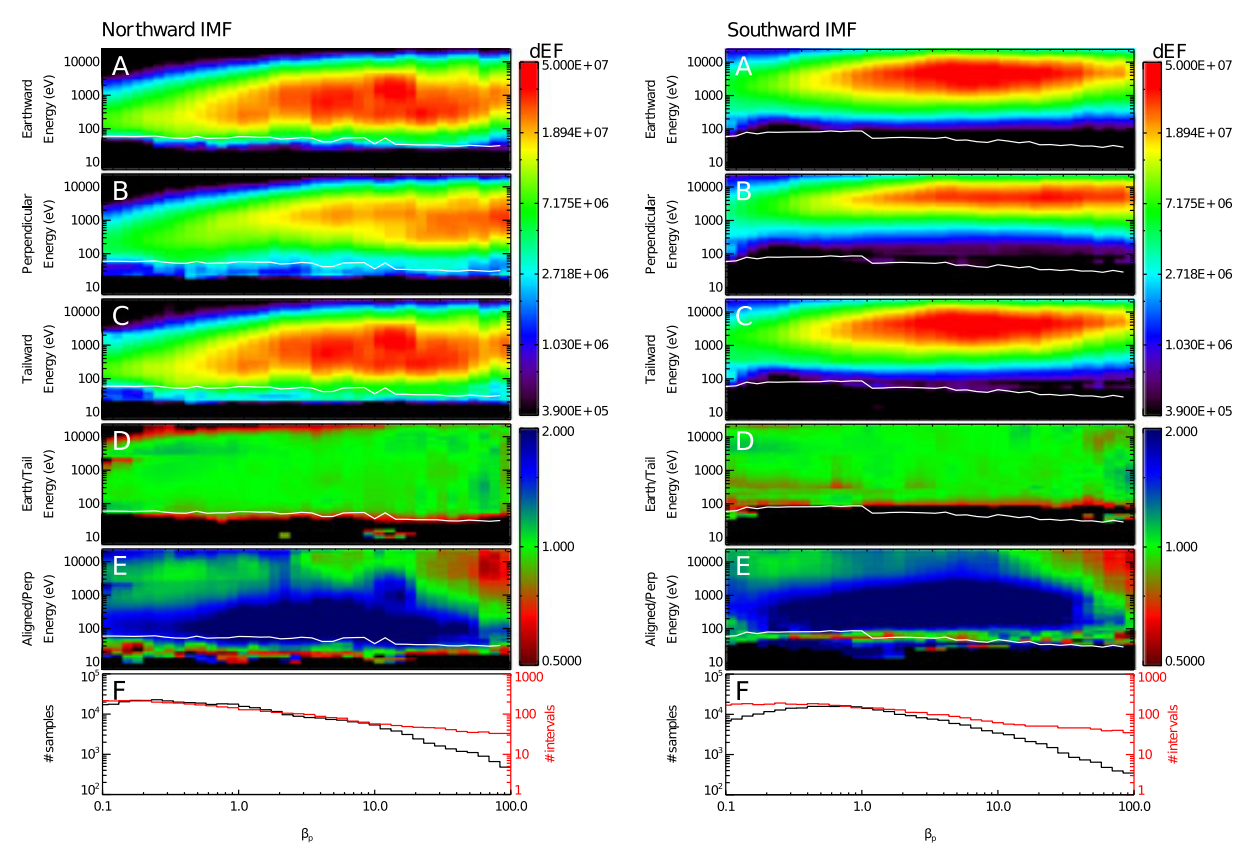

Figure 3. (a-f) The same as for Figure 1, but for the northward IMF data set (left) and the southward IMF data set (right).

(that of the electron pitch angle distributions) to allow for binning and comparison.

[10] Following Walsh et al. [2011], we further limited the data set to those times at which Cluster 4 was located further than $15 R_{E}$ from the Earth on the nightside, and when $0.01<n_{p}<10.0$. In doing so, we ensure our results are not confused by any radial evolution of pitch angle distributions; we eliminate those times when the count rates of protons are too low to produce reliable higher order moments [Rème et al., 2001] and we remove anomalous data spikes. Furthermore, those individual parts of the PADs (i.e., energy, angle, and time bins) where the count rate was at or below instrument background level were set to zero but still included, whereas those energy bins of the electron PADs below the instantaneous spacecraft potential, as measured by EFW and corrected according to the results of Cully et al. [2007], were disregarded. Where no spacecraft potential data were available, the average spacecraft potential for the concurrently measured proton density was used.

[11] The PADs were then rearranged to produce arrays containing particles flowing earthward parallel or antiparallel to the magnetic field (i.e., particles with a pitch angle of $0^{\circ}$ measured in the Northern Hemisphere and particles with pitch angles of $180^{\circ}$ measured in the Southern Hemisphere), particles flowing tailward parallel or antiparallel to the magnetic field (i.e., particles with a pitch angle of $180^{\circ}$ measured in the Northern Hemisphere and particles with pitch angles of $0^{\circ}$ measured in the Southern Hemisphere) and particles moving perpendicular to the magnetic field (pitch angle of $90^{\circ}$ ). These will henceforth be referred to as earthward, tailward, and perpendicular populations, respectively. Both the northward IMF and southward IMF data sets were then split into 50 evenly spaced bins based on instantaneous $\log _{10}\left(\beta_{p}\right)$, again following the procedure of Walsh et al. [2011], although here we used 50 bins rather 100 because of the comparatively small size of each subset. The mean electron $\mathrm{dEF}$ as a function of energy in the earthward, tailward, and perpendicular directions were then calculated for each $\beta_{p}$ bin, allowing us to compare the behavior of pitch angle distributions under constant IMF conditions with the results of Walsh et al. [2011], who did not take IMF direction into account.

[12] Note that the electron pitch angle distributions will be discussed in terms of differential energy flux and have been analyzed in the spacecraft frame. Because the PADs are produced from thin (within the limit of instrument angular resolution) slices of the full 3D velocity distribution that are selected on-board the spacecraft, transforming them from the spacecraft to the plasma frame is difficult; furthermore, the bulk velocity of the plasma only has a small effect on electron energy compared to instrument energy resolution, so the frame transformation would not produce a PAD significantly different from that which was measured. We also emphasize that data are taken from individual pitch angle bins $\left(15^{\circ}\right.$ in size $)$ and are not integrations of the pitch angle distributions over relevant angle ranges.

\section{Observations}

[13] The average pitch angle distributions of electrons observed in the magnetotail, binned by $\beta_{p}$, and plotted as a function of $\beta_{p}$ and energy for northward IMF and southward IMF are plotted in Figure 3 left and right, respectively, which follow the same format as Figure 1. Briefly, in both figures, earthward fluxes are plotted in Figure $3 a$, perpendicular fluxes in Figure $3 b$, and tailward fluxes in Figure 3c. The color scale represents differential energy flux. The ratio between earthward and tailward fluxes is plotted in Figure 3d. The ratio between the average field-aligned flux (earthward+tailward) $/ 2$ and perpendicular flux is plotted in Figure 3e. Thus, a green color in Figure $3 \mathrm{~d}$ and blue in Figure 3e signify an average electron 

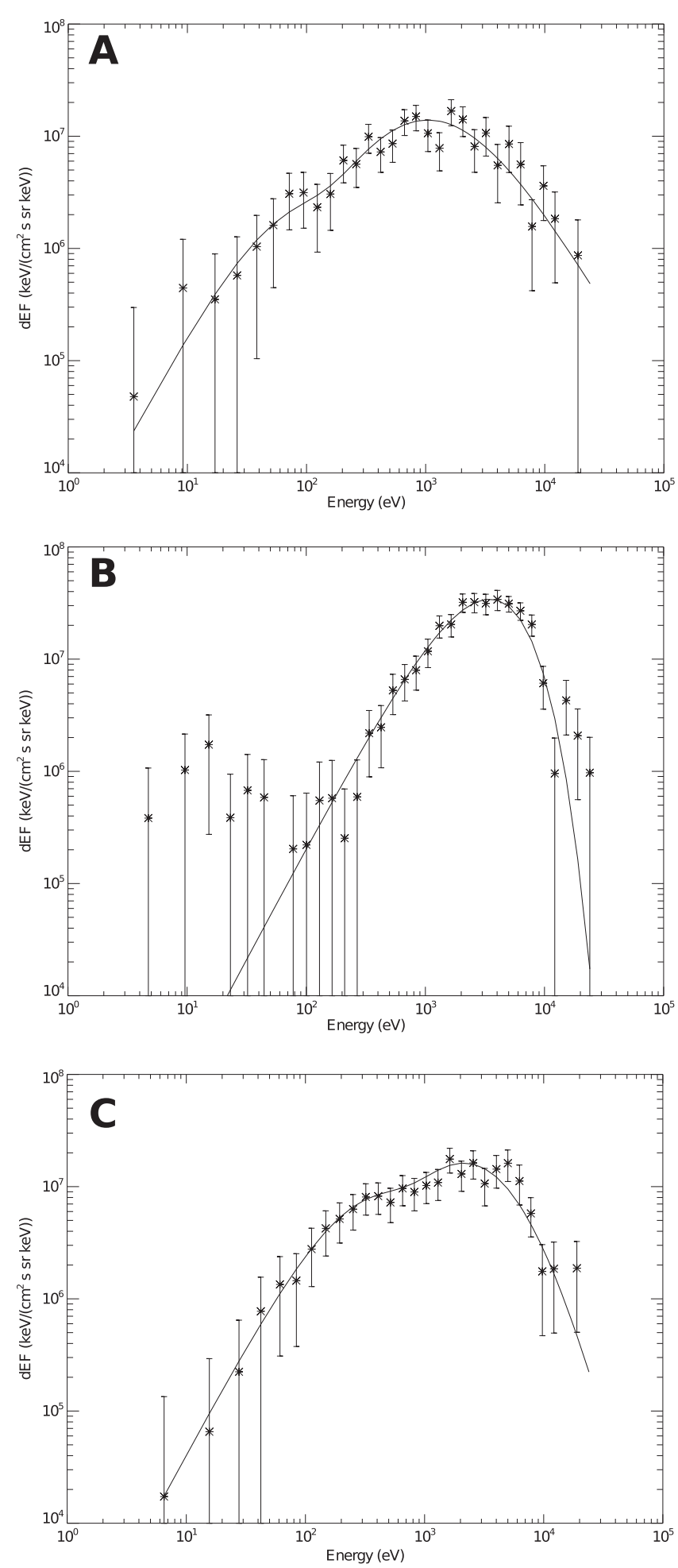

Figure 4. Example two-component fits that have been (a) accepted and (b) rejected according to the logarithmic $\chi^{2}$ criterion. Both of these fits would be rejected using linear $\chi^{2}$. (c) A fit that would be accepted by both is plotted. The solid lines are the fits, data are symbols. Error bars are calculated from the counting statistics. distribution dominated by bidirectional, field-aligned electron flux. White lines overplotted on the spectrograms are the maximum spacecraft potential observed in a particular bin, so electrons observed at energies above this line are unlikely to be photoelectrons. The number of individual PADs that make up the averages in each $\beta$ bin are plotted in black in Figure 3f, while the number of discrete intervals during which those distributions were measured is plotted in red.

[14] The main features of the average electron plasma sheet (Figure 1) are evident for during both steady northward (Figure 3, left) and steady southward (Figure 3, right) IMF:

[15] 1. Electron dEF increases with increasing $\beta_{p}$ for all directions, reaching a maximum where $\beta_{p} \geq 2-3$ (Figures 3a-3c).

[16] 2. Electron dEF above background is observed at increasingly high energies with increasing $\beta_{p}$ for all directions, although this is more obvious for northward IMF (Figures 3a-3c).

[17] 3. Earthward and tailward electron $\mathrm{dEF}$ are for the most part balanced, indicating the presence of bidirectional electrons (Figure 3d).

[18] 4. At sub-keV energies, field-aligned electron $\mathrm{dEF}$ is higher than perpendicular electron $\mathrm{dEF}$ for $\beta_{p} \leq 20$ (Figure 3e).

[19] 5. At $\beta_{p} \geq 20$, and super-keV energies, the PAD is dominated by perpendicular electrons (Figure $3 \mathrm{e}$ ).

[20] There are, however, also some differences between the average northward IMF and southward IMF PADs. Under southward IMF, the plasma sheet electrons typically have a higher energy than under northward IMF, so there is a higher dEF of electrons at the top of the PEACE energy range. While the PADs are dominated by bidirectional electrons at sub-keV energies for both northward and southward IMF, at the highest energies, there is a difference between the two sets of averages. For southward IMF (Figure 3e, right), for $0.7 \geq \beta_{p} \leq 10$, the bidirectional dominance extends to the top of the PEACE energy range, which is not the case for northward IMF (Figure 3e, left), where at the highest PEACE energies, the electron dEF is balanced. For northward IMF, there is an apparent net tailward flux of electrons at the top of the PEACE energy range (red in Figure 3d, left). This is an instrument effect caused by sunlight shining into the aperture of one of the PEACE sensors when its look direction is pointed toward the Sun, and hence measuring tailward flowing electrons. It is only a significant effect when there are low natural fluxes of electrons, which explains why it is not present in Figure 3 (right).

\section{Analysis}

[21] Each $\beta_{p}$ bin contains at least 300 (for the highest values of $\beta_{p}$ ), and more often $>3000$ individual PADs taken from at least 30 different intervals (Figure 3f) suggesting these results are robust. Indeed, the standard error on the mean for those $\beta_{p}$ and energy bins with electron flux above background ranges is usually $<4 \%$, comparable with the errors on the results of Walsh et al. [2011]. The existence of the pitch angle anisotropy in the average plasma sheet electron PADs at sub-keV energies is persistent, then, for both northward and southward IMF. This suggests that the results of Walsh et al. [2011] cannot be explained as a superposition 


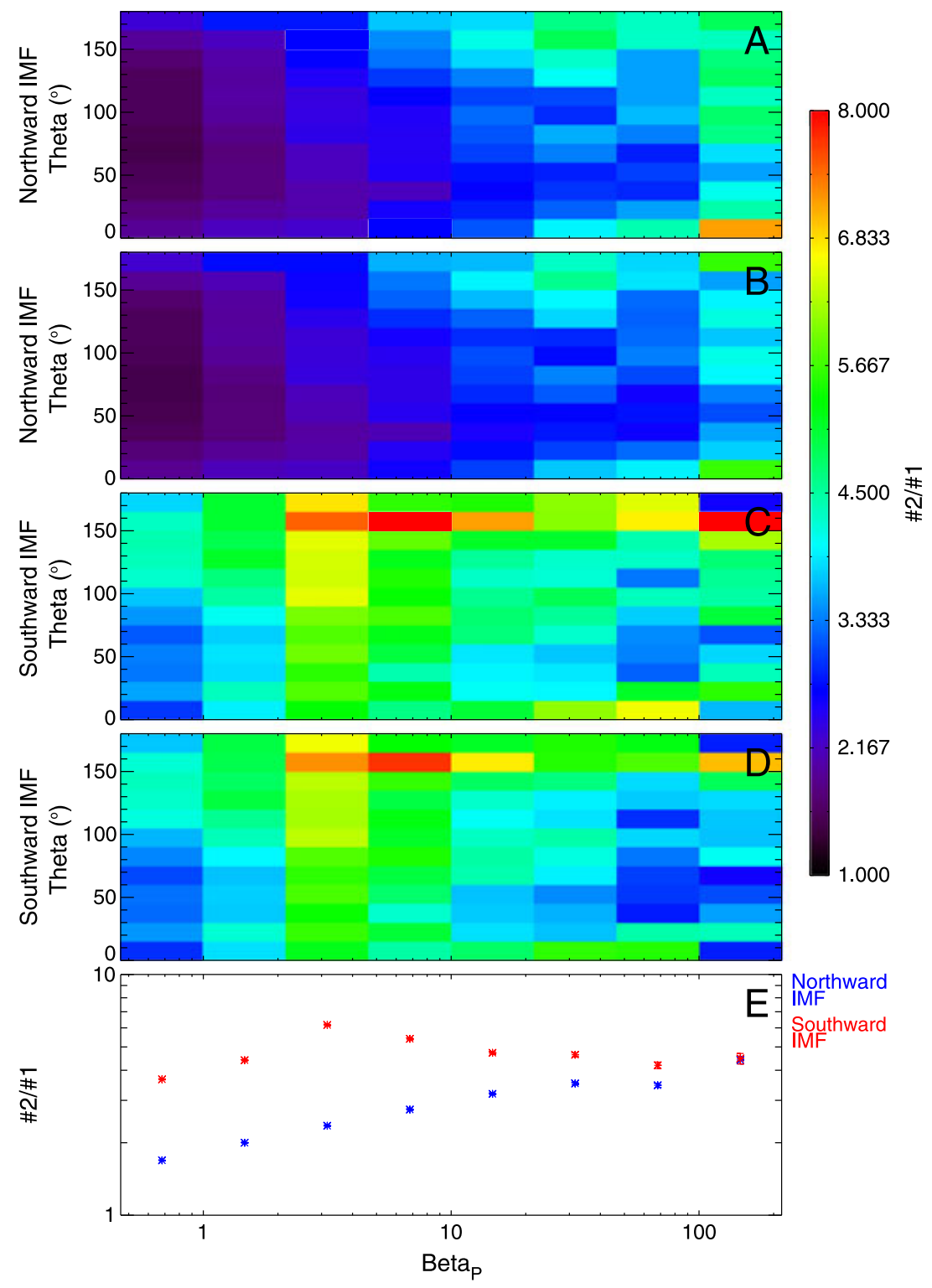

Figure 5. The occurrence ratio of two component fits to one component fits plotted as a function of $\theta$ and $\beta_{p}$. Electrons with $0^{\circ} \leq \theta<90^{\circ}$ have an earthward component of $v_{\|}$while electrons with $90^{\circ} \leq \theta \leq 180^{\circ}$ have a tailward component of $v_{\|}$. (a and c) Raw ratios are plotted in (a) and (c), while ratios taking into account uncertainty are plotted in (b) and (d). (e) The omnidirectional equivalent, not considering $\theta$.

of the plasma sheet in quiet and disturbed states controlled by IMF $B_{z}$. Another possibility is that the anisotropy is the result of the presence of a second, cold, component of electrons that has higher flux in the parallel and antiparallel directions. Indeed, it is well known that electrons in the terrestrial plasma sheet can be modeled using a twocomponent kappa distribution [Wang et al., 2007; Gabrielse et al., 2012] and two component kappa distributions also accurately describe the Saturnian plasma sheet [Schippers et al., 2008], suggesting that two component plasmas might be a common feature of planetary magnetospheres.

[22] To investigate the occurrence and properties of this cold component, we attempt to fit each electron distribution in either of the northward IMF or southward IMF data sets that was measured when $\beta_{p} \geq 0.1$ to both a single component (equation (1)) and two component kappa distribution (equation (2)), considering each pitch angle bin separately, i.e.,

$$
J_{E}=A E^{2}\left(\frac{1}{\kappa E_{0}}\right)^{\frac{3}{2}} \frac{\Gamma(\kappa+1)}{\Gamma\left(\kappa-\frac{1}{2}\right)}\left(1+\frac{E}{E_{0} \kappa}\right)^{-\kappa-1}
$$

and

$$
\begin{aligned}
J_{E}= & A_{c} E^{2}\left(\frac{1}{\kappa_{c} E_{0 c}}\right)^{\frac{3}{2}} \frac{\Gamma\left(\kappa_{c}+1\right)}{\Gamma\left(\kappa_{c}-\frac{1}{2}\right)}\left(1+\frac{E}{E_{0 c} \kappa_{c}}\right)^{-\kappa_{c}-1} \\
& +A_{h} E^{2}\left(\frac{1}{\kappa_{h} E_{0 h}}\right)^{\frac{3}{2}} \frac{\Gamma\left(\kappa_{h}+1\right)}{\Gamma\left(\kappa_{h}-\frac{1}{2}\right)}\left(1+\frac{E}{E_{0 h} \kappa_{h}}\right)^{-\kappa_{h}-1},
\end{aligned}
$$


Table 1. Number of Pitch Angle Distributions Included in the Survey and the Numbers of Successful One and Two Component Fits

\begin{tabular}{lcc}
\hline & Northward IMF & Southward IMF \\
\hline Whole data set $^{\mathrm{a}}$ & 391,079 & 283,353 \\
One component $^{\mathrm{b}}$ & 387,770 & 138,431 \\
Two component $^{\mathrm{b}}$ & 828,589 & 659,861 \\
\hline
\end{tabular}

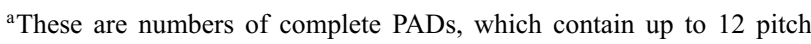
angle bins, each fit individually.

${ }^{\mathrm{b}}$ These are individual fits, i.e., $\sim 1 / 12$ of a PAD.

where $J_{E}$ is the differential energy flux, $A$ is a parameter proportional to density, $E$ is the electron energy, $E_{0}$ is the most likely energy of electron, and $\kappa$ is the kappa index, a measure of the slope of the suprathermal tail of the distri- bution. The subscripts $c$ and $h$ denote parameters associated with the cold and hot components of the two component distribution, respectively.

[23] This scalar, one-dimensional, formulation of the kappa distribution [cf. Haaland et al., 2010] is used because of the sometimes incomplete pitch angle coverage in the PEACE data (usually when the magnetic field direction is varying on timescales smaller than a spacecraft spin, $\sim 4 \mathrm{~s}$ ); it allowed us to fit each pitch angle direction independently and hence maximize the size of the available data set.

[24] Fitting was carried out using the LevenbergMarqwardt algorithm [Markwardt, 2009], taking $A, E_{0}$, and $\kappa$ to be free parameters. Individual PADs were corrected for spacecraft potential before fitting, and both the data and the model function were converted to counts/accumulation before evaluating the residuals; so the fits could be weighted by the error on the measurement taken to be the counting
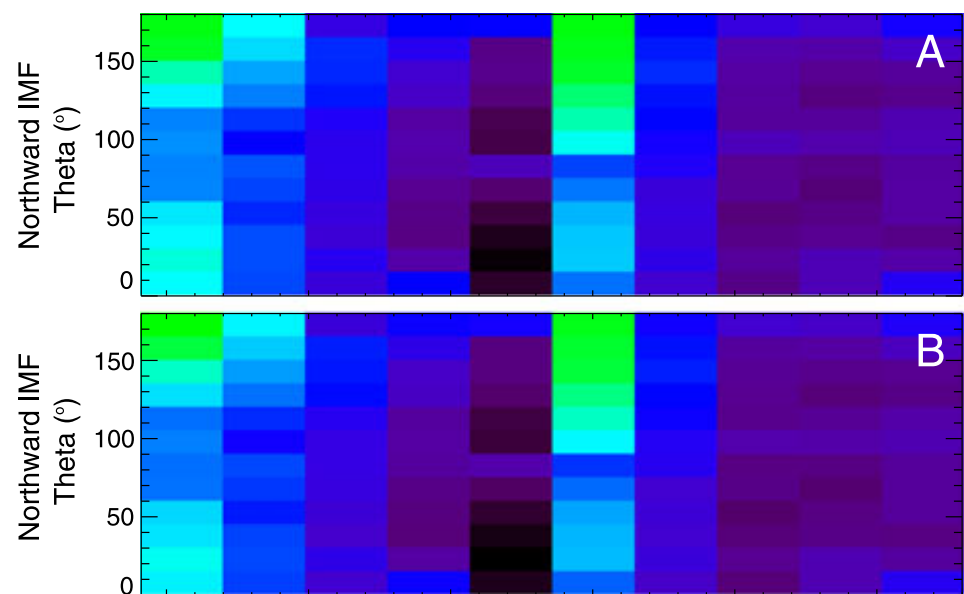

$-26.050$

B

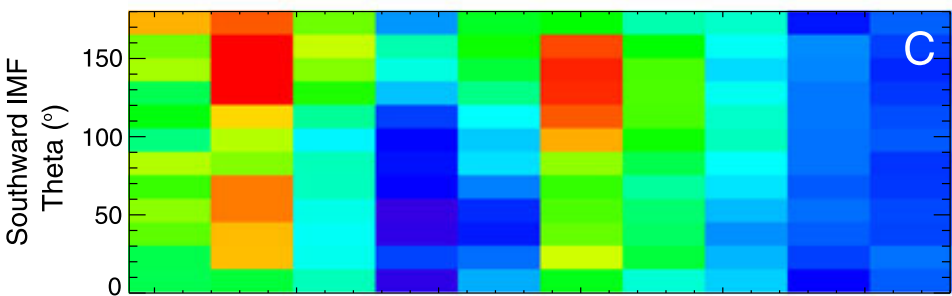

$7.071 \underset{\#}{\#}$
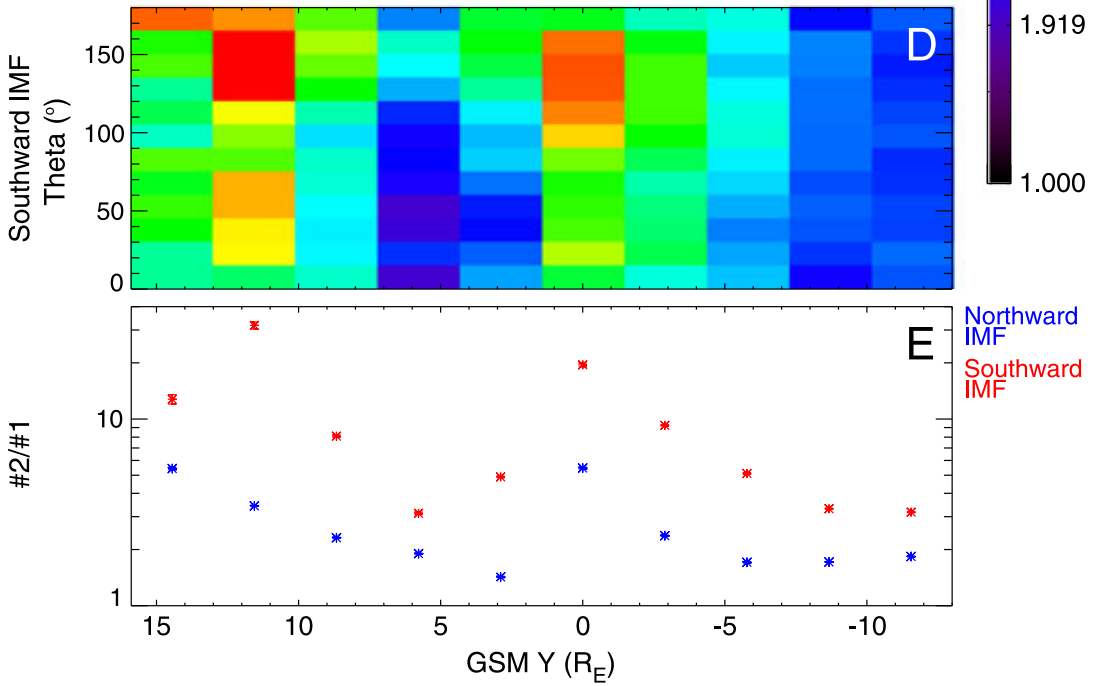

Figure 6. The same as for Figure 5, but plotted as a function of GSM $Y$ rather than $\beta_{p}$. 

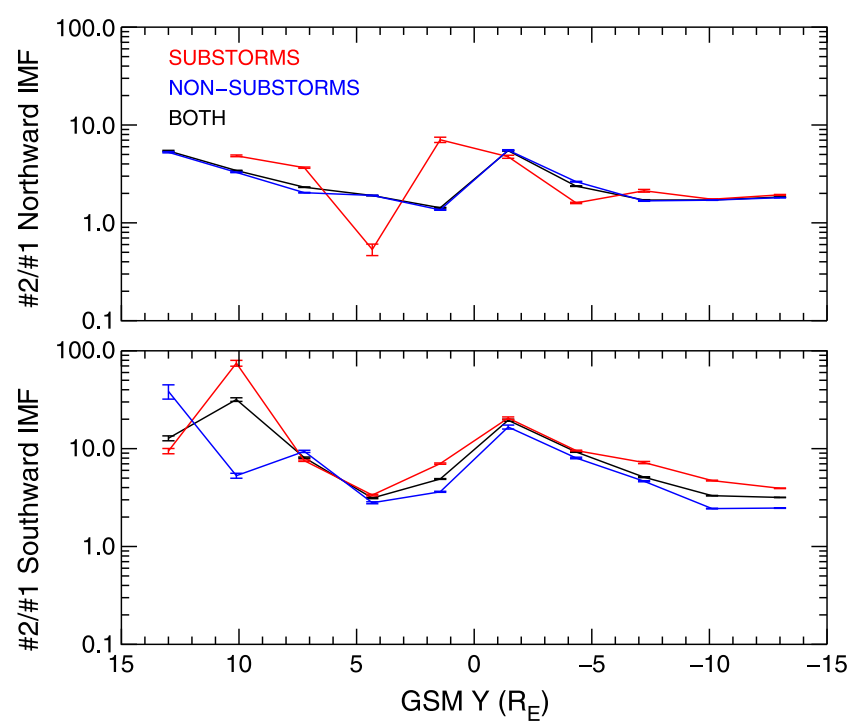

Figure 7. Occurrence ratio of two component to one component fits as a function of GSM $Y$ under (a) northward IMF and (b) southward IMF, split according to the level of substorm activity. Blue curves are for nonsubstorm intervals, red for substorm intervals.

statistics, i.e., $\sqrt{\text { counts/accumulation. }}$ A fit was judged to be successful when the $\chi^{2}$ value was less than the critical value for a $95 \%$ confidence level, although this was evaluated based on the natural logarithm of the data and model function. Count rates in a typical distribution range from $\sim 10$ to $\sim 10,000$ and the distributions are often noisy even at high count rates. Evaluating the $\chi^{2}$ value in linear space almost always resulted in fits that were significantly different from the data at the $95 \%$ confidence level, despite appearing to reproduce the data well when plotted on a log-log scale, as is typical. Using the natural logarithm to evaluate the goodness of fit has the result of excluding the poorest fits while including those that well reproduce the data as it is usually plotted. Examples of fits that are deemed unsuccessful using both linear and logarithmic $\chi^{2}$, deemed successful using logarithmic $\chi^{2}$ but unsuccessful using linear $\chi^{2}$, and deemed successful using both methods are plotted in Figure 4.

[25] The difference in $\chi^{2}$ between the one component kappa fit and two component kappa fit was used to determine which model distribution best fit the data. Here those energies for which the model counts/accumulation was within the error of the measured counts/accumulation were taken to have residuals of zero when calculating the $\chi^{2}$. If both the one component and two component fits were entirely within error of the data, both $\chi^{2}$ would be zero and that distribution was not counted as either a single component or two component distribution. If either $A_{c}$ or $A_{h}$ for the two component fit was zero, that distribution was counted as a single component rather than two component distribution.

[26] The numbers of one and two components fits for northward and southward IMF, as well as the total size of each data set (where $\beta_{p}>0.1$ ) are listed in Table 1 . Two component distribution fits are more often obtained than one component distribution fits for both northward and southward IMF. The ratio between the number of two component and one component distributions is plotted as a function of $\beta_{p}$ in Figure 5. The ratio is plotted as a function of both $\beta_{p}$ and the angle $\theta$ in Figures $5 \mathrm{a}-5 \mathrm{~d}$. The angle $\theta$ is the pitch angle rearranged by hemisphere, i.e., $0^{\circ} \leq \theta<$ $90^{\circ}$ signifies electrons with an earthward component of $v_{\|}$, while $90^{\circ}<\theta \leq 180^{\circ}$ signifies electrons with a tailward component of $v_{\|}$, regardless of the magnetic field direction. Figures $5 \mathrm{a}$ and $5 \mathrm{~b}$ are for northward IMF, and Figures $5 \mathrm{c}$ and $5 \mathrm{~d}$ are for southward IMF. Figures $5 \mathrm{a}$ and $5 \mathrm{c}$ are simply the ratio of the number of two component fits to the number of one component fits observed in a given $\left(\beta_{p}, \theta\right)$ bin while the ratios plotted in Figures $5 \mathrm{~b}$ and $5 \mathrm{~d}$ take into account counting statistics, adding the Poisson error to the number of one component fits and subtracting it from the number of two component fits thus pushing the ratio as close to unity as the uncertainty allows. A comparison of Figures $5 \mathrm{a}$ with $5 \mathrm{~b}$ and $5 \mathrm{c}$ with $5 \mathrm{~d}$ shows that the data set is sufficiently large that counting statistics do not greatly alter the ratios. Figure $5 \mathrm{e}$ is the ratio between the total number of two component and total number of one component fits for all values of $\theta$, i.e., not taking into account direction, for northward IMF (blue) and southward IMF (red). Error bars are the uncertainty on the ratio, again derived by propagating the counting statistics for each bin, and are typically similar in size to the plotting symbol.

[27] The ratio between the number of two component and the number of one component fits is always above one, i.e., it is always more likely that a two component fit is obtained than a one component fit. For northward IMF, the ratio increases with increasing $\beta_{p}$ (Figures $5 \mathrm{a}, 5 \mathrm{~b}$, and $5 \mathrm{e}$ ) and is higher for tailward-moving electrons than for perpendicularor earthward-moving electrons (Figures $5 \mathrm{a}$ and $5 \mathrm{~b}$ ). For southward IMF, the ratio is generally higher than for northward IMF and exhibits similar behavior in terms of $\theta-$ tailward-moving electrons are again more likely to be identified as having two components than perpendicular- or earthward-moving electrons. The behavior as a function $\beta_{p}$ is more complex than for northward IMF. The ratio peaks for $3.0 \leq \beta_{p} \leq 10$ (Figures $5 \mathrm{c}-5 \mathrm{e}$ ) and is equal to the northward IMF ratio for the highest $\beta_{p}$ (Figure 5e).

[28] The occurrence ratio is plotted as a function of GSM $Y$ in Figure 6, which follows the same format as Figure 5. The ratio is strongly organized by $Y$ for both northward (Figures 6a, 6b, and 6e) and southward (Figures 6c-6e) IMF: There is a peak in occurrence ratio just dawnward of the noon-midnight meridian and a further peak toward the dusk. Similarly to in Figure 5, tailward-moving electrons have the highest likelihood of being better described by a two component distribution than a one component distribution.

[29] The occurrence ratio for all $\theta$ is also plotted as a function of GSM $Y$ in Figure 7, although here the data set is split into substorm and nonsubstorm intervals. For a distribution to be included in the substorm subset, it must have been measured either less than 30 min prior to a substorm onset or less than $2 \mathrm{~h}$ after a substorm onset; conversely, for a distribution to be included in the nonsubstorm intervals, it must have been measured more than $30 \mathrm{~min}$ prior to any onset and more than $2 \mathrm{~h}$ after any onset. Substorm onset times were taken from the Supermag substorm onset list [Newell and Gjerloev, 2011a, 2011b] and the time criteria were based on the median substorm phase durations reported 

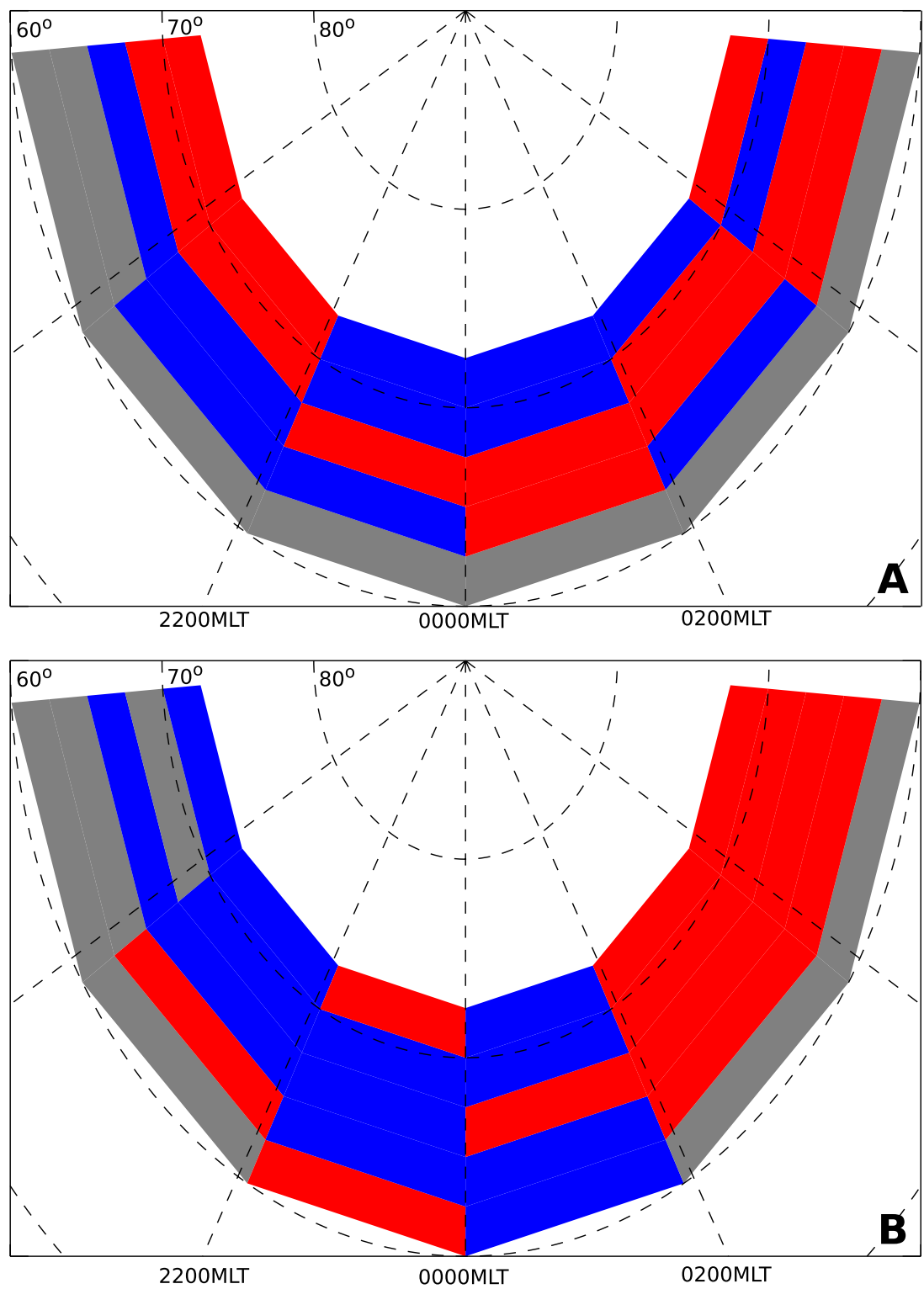

Figure 8. Occurrence ratio for $\theta=180^{\circ}$ (i.e., tailward field-aligned) electrons mapped to the ionosphere for (a) northward IMF and (b) southward IMF. Red-shaded cells are those MLAT/MLT bins where the ratio is below the median for that data set, blue-shaded cells are those bins where the ratio is above the median. Gray cells are those where there are fewer than 10 one or two component fits making up the ratio.

by Juusola et al. [2011]. Error bars are calculated as in Figures 5 and 6.

[30] Substorm activity does not have a large effect on the two to one component fit occurrence ratio for southward IMF, although it is slightly higher during substorm intervals than nonsubstorm intervals (Figure 7b). For northward IMF (Figure 7a), the occurrence ratio during substorm intervals is an exaggerated version of the occurrence ratio during nonsubstorm intervals - the peak just dawnward of the noon-midnight meridian is larger and broader, extending farther in $Y$, while the decrease duskward of this peak is also of greater amplitude - decreasing below one. Substorms are sufficiently few, however, that the overall occurrence ratio is almost identical to the ratio for nonsubstorm intervals, lending credibility to our IMF selection criteria.
[31] The two to one component fit occurrence ratio as a function of the magnetic latitude (MLAT; in Altitude Adjusted Corrected Geomagnetic Coordinates) and magnetic local time (MLT) of the spacecraft foot point is plotted for northward and southward IMF in Figures $8 \mathrm{a}$ and $8 \mathrm{~b}$, respectively. For simplicity, and to ensure availability of input parameters for all distributions, mapping was carried out using the Tsyganenko [1989] magnetic field model. Here only $\theta=180^{\circ}$ electrons are considered, i.e., those tailward-moving electrons that are the most field-aligned. Foot points were mapped to the nearest ionosphere to avoid tracing through the tail current sheet and the absolute value of MLAT was taken for the purposes of binning. Those MLT/MLAT bins where the occurrence ratio is larger than the median occurrence ratio are colored blue, while those 

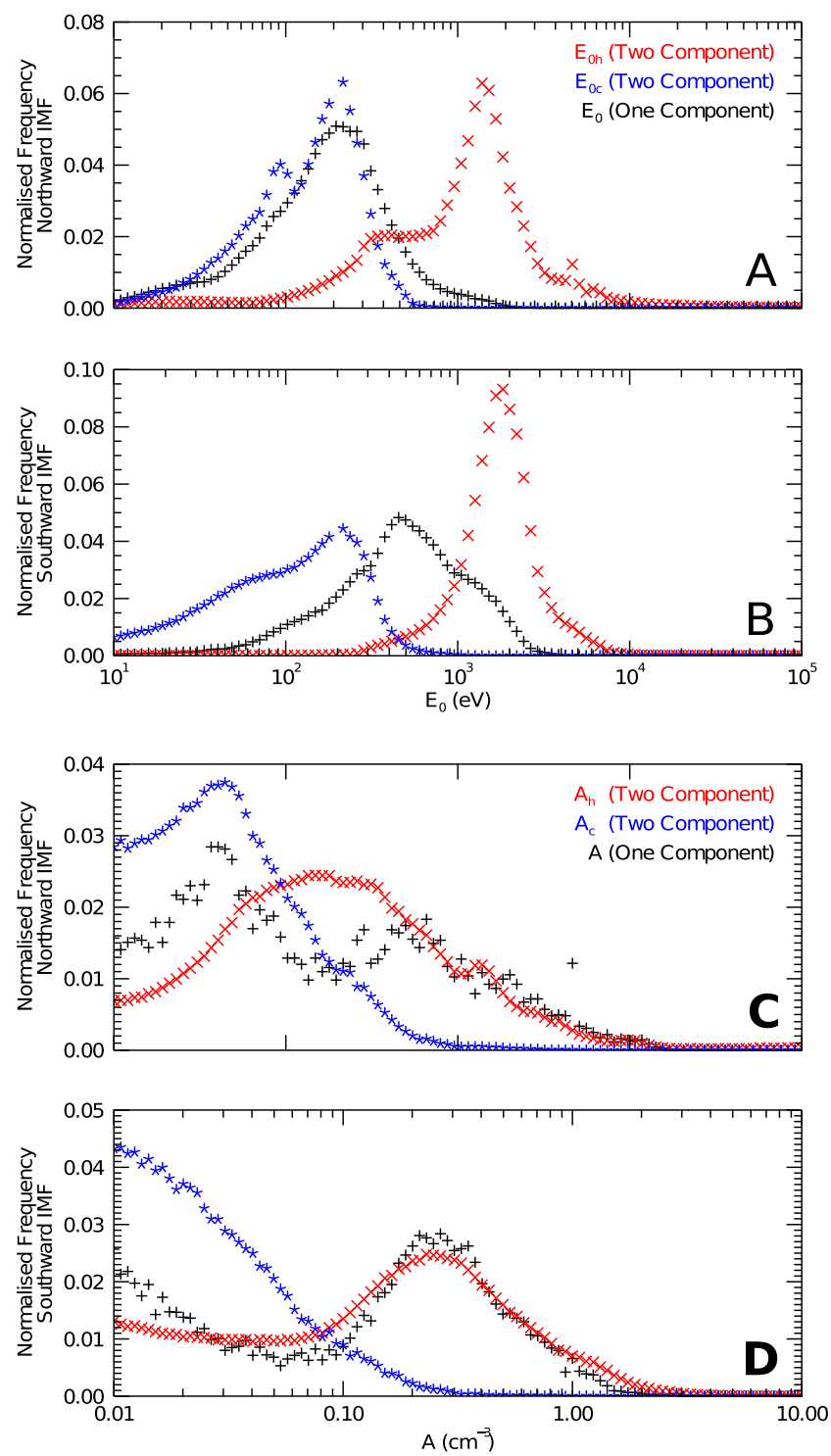

Figure 9. Normalized histograms of $E_{0}$ values for (a) northward and (b) southward IMF. Black curves are the one component $E_{0}$, while blue and red curves are the two component $E_{0 c}$ and $E_{0 h}$, respectively. (c and d) Equivalent plots for fit densities $\left(A, A_{c}\right.$, and $A_{h}$ are plotted.

bins where the occurrence ratio is smaller than the median are colored red. Gray-shaded bins are those in which there are fewer than 10 one component or two component fits. For northward IMF, duskward of the noon-midnight meridian, the bins with the highest occurrence ratios tend to be at lower latitudes than the bins with the lower occurrence ratios, while the converse is true for those bins dawnward of the noon-midnight meridian. The pattern for southward IMF (Figure 8b) exhibits more of a straightforward dawndusk asymmetry, where the bins with an occurrence ratio higher than the median are concentrated mainly in the premidnight sector.

[32] The distribution of the values of the three $E_{0}$ parameters, i.e., $E_{0}$ (one component), and $E_{0 c}$ and $E_{0 h}$ (two component) for northward and southward IMF are plotted in
Figures $9 \mathrm{a}$ and $9 \mathrm{~b}$, respectively. $E_{0}$ is plotted in black, $E_{0 c}$ in blue and $E_{0 h}$ in red. Those fits where the fitting procedure did not alter $E_{0}$ from its starting value are not included in the figure and each curve is normalized by the total number of successful fits. This figure takes into account all directions. The peak value of $E_{0 c}$ for both northward and southward IMF is around $200 \mathrm{eV}$, with a secondary peak around $70 \mathrm{eV}$, which is more pronounced for northward IMF than southward. $E_{0 h}$ is peaked between $1000 \mathrm{eV}$ and $2000 \mathrm{eV}$, higher for southward IMF, consistent with the data (cf. Figure 3 left and right). Similarly, the distribution of fit densities, $A$, $A_{c}$, and $A_{h}$, are plotted in Figures 9c and 9d. Typically, the hot component has a higher fit density than the cold component, although this difference is reduced for northward IMF. The kappa parameter is lowest for the single component fits, while for the two component fits, $\kappa_{c}$ is lower than $\kappa_{h}$. The values are $2.89,12.8$, and 21.9 , respectively, for northward IMF and 3.40, 5.07, and 24.3, respectively, for southward IMF.

\section{Discussion}

[33] Our observations show that the anisotropy in magnetotail electron pitch angle distributions noted by Walsh et al. [2011] can be explained as the result of a cold component of electrons that is present under both northward and southward IMF conditions (Figure 3). The cold component is most likely observed in tailward-moving electrons (Figure 5) and exists mainly at sub-keV energies (Figure 9). For northward IMF, the cold component is more often observed on field lines that map to lower latitudes in the premidnight sector and higher latitudes in the postmidnight sector, while for southward IMF, the cold component is more often observed in the premidnight sector than the postmidnight sector, regardless of latitude (Figure 8). It should be noted that an increased occurrence of successful two component fits to tailward flowing electron distributions does not necessarily imply that the average flux of electrons should be higher. The data show the average flux tends to be balanced (Figure $3 \mathrm{~d}$ ). It is possible that interactions with the magnetotail current sheet will modify the electron distribution such that it is less likely to be successfully fitted with a two component kappa, without, on average, altering the flux. The number of successful tailward fits compared to earthward fits is consistent with this: There are $7 \%$ more successful tailward fits than earthward fits for northward IMF. The equivalent number for southward IMF is $10 \%$.

[34] While the cold electrons in the magnetotail plasma sheet have not been well studied, the presence of a cold component of ions in the magnetotail plasma sheet has been well established [e.g., Terasawa et al., 1997; Fujimoto et al., 1998; Øieroset et al., 2005; Wing et al., 2005; Nishino et al., $2007 \mathrm{~b}$ ]. Cold ions are more often observed under northward IMF than southward IMF [Øieroset et al., 2005; Wing et al., 2005], to the extent that a separate cold component is almost exclusively observed under northward IMF [Nishino et al., 2007b, 2007c]. Transport through the flank magnetopause, perhaps via reconnection in rolled-up Kelvin-Helmholtz vortices [Hasegawa et al., 2004], is thought to be responsible for the presence of cold ions in the magnetotail under northward IMF. Cold electrons with a dominant parallel component have been observed contemporaneously with the 
two component ion plasma sheet [Nishino et al., 2007a] and indeed close to a Kelvin-Helmholtz vortex [Nishino et al., 2007], so it is possible that the persistent cold component reported here also has a magnetosheath source.

[35] Our results, however, are more consistent with an ionospheric source for cold electrons than a magnetosheath source. The cold component of electrons is more often observed under southward IMF than northward IMF (Figures 5 and 6), whereas Kelvin-Helmholtz driven transport is known to dominate under northward IMF [Otto and Fairfield, 2000; Nykyri and Otto, 2001; Hasegawa et al., 2006]. There is a peak in occurrence of the cold component of electrons at dusk (Figure 6), similar to that observed for protons [Nishino et al., 2007b], which is consistent with a magnetosheath source. However, if the cold electrons did have a magnetosheath source, a peak toward dawn should also be present [Wing et al., 2005] and this is not observed. Furthermore, the distribution of $E_{0 c}$ (Figure 9) is similar for northward and southward IMF, implying that the same acceleration or transport mechanism operates under both circumstances. Contrast this with the behavior of $E_{0 h}$, which is typically higher under southward IMF than northward IMF suggesting an acceleration or heating process that occurs more often under southward IMF, consistent with the results of Christon et al. [1989, 1991].

[36] The increased likelihood of observing the cold component of electrons during substorms (Figure 7) is also consistent with an ionospheric source. Cold electrons have been observed within the near-Earth plasma sheet just prior to substorm onset and have been linked to field-aligned current systems connecting the magnetosphere to the ionosphere [Walsh et al., 2010]. The locations in the ionosphere to which $\mathrm{C} 4$ mapped (Figure 8) when most often observing the cold component of electrons traveling tailward along the magnetic field direction are also consistent with this interpretation. Under northward IMF (Figure 8a), the observed pattern is similar to that of the large-scale downward fieldaligned currents derived during quiet intervals from Triad magnetometer data by Iijima and Potemra [1978], even to the extent that a feature resembling the Harang Discontinuity is observed just duskward of midnight in Figure 8a. Downward current systems have been observed in the past to be associated with upwelling electrons at low altitude [Carlson et al., 1998], albeit with slightly higher energies than typically observed here, and in the high-latitude plasma sheet at a distance of 5-7 $R_{E}$ [Kletzing and Scudder, 1999]. Cold, field-aligned electrons have also been observed in the equatorial plasma sheet by Cluster, conjugate with fast observations of a downward current region and the associated electron beam [Wright et al., 2008]. It has been established, then, that electrons from the ionosphere can reach the equatorial plasma sheet. Our results show that they do so often enough to be observed as a persistent feature of the plasma sheet even when the magnetosphere is undisturbed. Our results also suggest that the large-scale field-aligned currents connecting the magnetosphere and ionosphere play a role in transporting the electrons from the ionosphere to the plasma sheet at quiet times.

[37] Under southward IMF, the pattern of large-scale field-aligned currents cannot be reconstructed from the plasma sheet electron observations; instead, a more straightforward dawn-dusk asymmetry is observed (Figure 8b).
This could simply be a result of less precise magnetic field mapping under more disturbed conditions [e.g., Kubyshkina et al., 2011] masking and distorting the pattern; because of the presence of additional current systems under southward IMF [cf. Wright et al., 2008] that do not contribute significantly under northward IMF, or indeed a combination of the two. The sense of the dawn-dusk asymmetry in occurrence is the same as other measures of magnetotail dynamics, for example, the thickness of the current sheet [Runov et al., 2005, 2006] and the duskward offset of the magnetotail $X$ line [Imber et al., 2011].

[38] While we have referred to the cold component as the second component, it is not clear whether or not there is a primary or preferred component for a given IMF direction. Under southward IMF, the dual component plasma sheet is identified more often and, when present, the $E_{0}$ distribution for the single component shows no preference toward the hot or cold plasma sheets (Figure 9b). In contrast, under northward IMF, the $E_{0}$ distribution for the single component plasma sheet is similar to the distribution of $E_{0 c}$ for the dual component plasma sheet (Figure 9A). Given that the cold dense plasma sheet is reported for northward IMF and thought to form through the entry of cold plasma into the tail [e.g., Fujimoto et al., 1998; Nishino et al., 2007a], our results could be interpreted as showing that the hot population is generally present in the plasma sheet but the cold component increases with increasing duration of northward IMF. The distributions of the $A$ parameters are consistent with this: The modal $A_{c}$ for northward IMF is higher than that for southward IMF, while the modal $A_{h}$ for northward IMF is lower than that for southward IMF (Figures 9c and 9d). This could, in principle, be tested further in a future study, but this is nontrivial given that one must account for plasma transport through the tail and is thus beyond the scope of this paper.

\section{Conclusions}

[39] We have examined electron pitch angle distributions, measured in the magnetotail plasma sheet by the Cluster PEACE instrument; during intervals, the magnetosphere has been exposed to steady northward and steady southward IMF. We find a cold component of electrons that is present the majority of the time and produces a pitch angle anisotropy such that parallel differential energy flux dominates at sub-keV energies. We present evidence that suggests that the source of these cold electrons is the ionosphere, and that when the magnetosphere is exposed to steady northward IMF, they are transported to the plasma sheet via the large-scale field-aligned current systems that couple the magnetosphere and ionosphere. Under southward IMF, the cold component electrons are observed more often and their occurrence pattern exhibits a more straightforward dawn-dusk asymmetry, with the two component fits obtained more often for measurements made in the premidnight sector. There is no obvious link a specific source in the ionosphere, or transport mechanism, for the southward IMF case.

[40] Acknowledgments. A.P.W. holds an ESA Fellowship. A.N.F., C.F., and C.J.O. are funded by UK STFC grant ST/H00260X/1. We acknowledge the Cluster instrument teams and Cluster Active Archive for provision of the Cluster data. We also acknowledge NASA/GSFC's Space 
Physics Data Facility's CDAWeb service for provision of OMNI solar wind data and the World Data Center for Geomagnetism, Kyoto for provision of kp data. We acknowledge SuperMAG and its constituent magnetometer networks for providing the substorm onset list. Portions of this work were carried out at the International Space Science Institute, Berne, Switzerland.

[41] Masaki Fujimoto thanks Iannis Dandouras and an anonymous reviewer for their assistance in evaluating this paper.

\section{References}

Balogh, A., et al. (2001), The Cluster magnetic field investigation: Overview of in-flight performance and initial results, Ann. Geophys., 19, 1207-1217.

Baumjohann, W. (1993), The near-Earth plasma sheet: An AMPTE/IRM perspective, Space Sci. Rev., 64, 141-163, doi:10.1007/BF00819660.

Carlson, C. W., et al. (1998), FAST observations in the downward auroral current region: Energetic upgoing electron beams, parallel potential drops, and ion heating, Geophys. Res. Lett., 25(12), 2017-2020, doi:10.1029/98GL00851.

Christon, S. P., D. J. Williams, D. G. Mitchell, L. A. Frank, and C. Y. Huang (1989), Spectral characteristics of plasma sheet ion and electron populations during undisturbed geomagnetic conditions, J. Geophys. Res., 94, 13,409-13,424, doi:10.1029/JA094iA10p13409.

Christon, S. P., D. J. Williams, D. G. Mitchell, C. Y. Huang, and L. A. Frank (1991), Spectral characteristics of plasma sheet ion and electron populations during disturbed geomagnetic conditions, J. Geophys. Res., 96, 1-22, doi:10.1029/90JA01633.

Cully, C. M., R. E. Ergun, and A. I. Eriksson (2007), Electrostatic structure around spacecraft in tenuous plasmas, J. Geophys. Res., 112, A09211, doi:10.1029/2007JA012269.

Eastman, T. E., L. A. Frank, W. K. Peterson, and W. Lennartsson (1984), The plasma sheet boundary layer, J. Geophys. Res., 89, 1553-1572, doi:10.1029/JA089iA03p01553.

Fujimoto, M., T. Terasawa, T. Mukai, Y. Saito, T. Yamamoto, and S. Kokubun (1998), Plasma entry from the flanks of the near-Earth magnetotail: Geotail observations, J. Geophys. Res., 103, 4391-4408, doi:10.1029/97JA03340.

Gabrielse, C., V. Angelopoulos, A. Runov, and D. L. Turner (2012), The effects of transient, localized electric fields on equatorial electron acceleration and transport toward the inner magnetosphere, J. Geophys. Res., 117, A10213, doi:10.1029/2012JA017873.

Gustafsson, G., et al. (2001), First results of electric field and density observations by Cluster EFW based on initial months of operation, Ann. Geophys., 19, 1219-1240.

Haaland, S., E. A. Kronberg, P. W. Daly, M. Fränz, L. Degener, E. Georgescu, and I. Dandouras (2010), Spectral characteristics of protons in the Earth's plasmasheet: Statistical results from Cluster CIS and RAPID, Ann. Geophys., 28(8), 1483-1498, doi:10.5194/angeo28-1483-2010.

Hasegawa, H., M. Fujimoto, T.-D. Phan, H. Rème, A. Balogh, M. W. Dunlop, C. Hashimoto, and R. Tandokoro (2004), Transport of solar wind into Earth's magnetosphere through rolled-up Kelvin-Helmholtz vortices., Nature, 430(7001), 755-8, doi:10.1038/nature02799.

Hasegawa, H., M. Fujimoto, K. Takagi, Y. Saito, T. Mukai, and H. RèMe (2006), Single-spacecraft detection of rolled-up Kelvin-Helmholtz vortices at the flank magnetopause, J. Geophys. Res., 111, A09203, doi:10.1029/2006JA011728.

Iijima, T., and T. A. Potemra (1978), Large-scale characteristics of fieldaligned currents associated with substorms, J. Geophys. Res., 83(A2), 599-615, doi:10.1029/JA083iA02p00599.

Imber, S. M., J. A. Slavin, H. U. Auster, and V. Angelopoulos (2011), A THEMIS survey of flux ropes and traveling compression regions: Location of the near-Earth reconnection site during solar minimum, $J$. Geophys. Res., 116, A02201, doi:10.1029/2010JA016026.

Johnstone, A. D., et al. (1997), PEACE: A plasma electron and current experiment, Space Sci. Rev., 79, 351-398.

Juusola, L., N. ØStgaard, E. Tanskanen, N. Partamies, and K. Snekvik (2011), Earthward plasma sheet flows during substorm phases, J. Geophys. Res., 116, A10228, doi:10.1029/2011JA016852.

Kletzing, C. A., and J. D. Scudder (1999), Auroral-plasma sheet electron anisotropy, Geophys. Res. Lett., 26(7), 971-974, doi:10.1029/ 1999GL900092.

Kubyshkina, M., V. Sergeev, N. Tsyganenko, V. Angelopoulos, A. Runov, E. Donovan, H. Singer, U. Auster, and W. Baumjohann (2011), Time-dependent magnetospheric configuration and breakup mapping during a substorm, J. Geophys. Res., 116, A00I27, doi:10.1029/ 2010JA015882.

Markwardt, C. B. (2009), Non-linear least-squares fitting in IDL with MPFIT, in Astronomical Data Analysis Software and Systems XVIII,
Astronomical Society of the Pacific Conference Series, edited by D. A. Bohlender, D. Durand, and P. Dowler, p. 251, vol. 411, Astronomical Society of the Pacific, San Francisco, Calif.

Newell, P. T., and J. W. Gjerloev (2011a), Evaluation of SuperMAG auroral electrojet indices as indicators of substorms and auroral power, J. Geophys. Res., 116, A12211, doi:10.1029/2011JA016779.

Newell, P. T., and J. W. Gjerloev (2011b), Substorm and magnetosphere characteristic scales inferred from the SuperMAG auroral electrojet indices, J. Geophys. Res., 116, A12232, doi:10.1029/ 2011JA016936.

Nishida, A. (2000), The Earth's dynamic magnetotail, Space Sci. Rev., 91, 507-577, doi:10.1023/A:1005223124330.

Nishino, M. N., M. Fujimoto, T. Terasawa, G. Ueno, K. Maezawa, T. Mukai, and Y. Saito (2007a), Temperature anisotropies of electrons and two-component protons in the dusk plasma sheet, Ann. Geophys., 25, 1417-1432, doi:10.5194/angeo-25-1417-2007.

Nishino, M. N., M. Fujimoto, T. Terasawa, G. Ueno, K. Maezawa, T. Mukai, and Y. Saito (2007b), Geotail observations of temperature anisotropy of the two-component protons in the dusk plasma sheet, Ann. Geophys., 25, 769-777, doi:10.5194/angeo-25-769-2007.

Nishino, M. N., M. Fujimoto, G. Ueno, K. Maezawa, T. Mukai, and Y. Saito $(2007 \mathrm{c})$, Geotail observations of two-component protons in the midnight plasma sheet, Ann. Geophys., 25, 2229-2245, doi:10.5194/angeo-252229-2007.

Nishino, M. N., M. Fujimoto, G. Ueno, T. Mukai, and Y. Saito (2007), Origin of temperature anisotropies in the cold plasma sheet: Geotail observations around the Kelvin-Helmholtz vortices, Ann. Geophys., 25, 2069-2086, doi:10.5194/angeo-25-2069-2007.

Nykyri, K., and A. Otto (2001), Plasma transport at the magnetospheric boundary due to reconnection in Kelvin-Helmholtz vortices, Geophys. Res. Lett., 28, 3565-3568, doi:10.1029/2001GL013239.

Øieroset, M., J. Raeder, T. D. Phan, T. D. Wing, J. P. McFadden, W. Li, M. Fujimoto, H. Rème, and A. Balogh (2005), Global cooling and densification of the plasma sheet during an extended period of purely northward IMF on October 22-24, 2003, Geophys. Res. Lett., 321, L12S07, doi:10.1029/2004GL021523.

Otto, A., and D. H. Fairfield (2000), Kelvin-Helmholtz instability at the magnetotail boundary: MHD simulation and comparison with geotail observations, J. Geophys. Res., 105, 21,175-21,190, doi:10.1029/ 1999JA000312.

Parks, G. K., M. McCarthy, R. J. Fitzenreiter, K. W. Ogilvie, J. Etcheto, K. A. Anderson, R. P. Lin, R. R. Anderson, T. E. Eastman, and L. A. Frank (1984), Particle and field characteristics of the highlatitude plasma sheet boundary layer, J. Geophys. Res., 89, 8885-8906, doi:10.1029/JA089iA10p08885.

Rème, H., et al. (2001), First multispacecraft ion measurements in and near the Earth's magnetosphere with the identical Cluster ion spectrometry (CIS) experiment, Ann. Geophys., 19, 1303-1354.

Runov, A., et al. (2005), Electric current and magnetic field geometry in flapping magnetotail current sheets, Ann. Geophys., 23, 1391-1403, doi:10.5194/angeo-23-1391-2005.

Runov, A., et al. (2006), Local structure of the magnetotail current sheet: 2001 Cluster observations, Ann. Geophys., 24, 247-262, doi:10.5194/ angeo-24-247-2006.

Schippers, P., et al. (2008), Multi-instrument analysis of electron populations in Saturn's magnetosphere, J. Geophys. Res., 113, A07208, doi:10.1029/2008JA013098.

Sergeev, V. A., E. M. Sazhina, N. A. Tsyganenko, J. A. Lundblad, and F. Soraas (1983), Pitch-angle scattering of energetic protons in the magnetotail current sheet as the dominant source of their isotropic precipitation into the nightside ionosphere, Planet. Space Sci., 31, 1147-1155.

Terasawa, T., et al. (1997), Solar wind control of density and temperature in the near-Earth plasma sheet: WIND/GEOTAIL collaboration, Geophys. Res. Lett., 24, 935-938, doi:10.1029/96GL04018.

Tsyganenko, N. A. (1982), Pitch-angle scattering of energetic particles in the current sheet of the magnetospheric tail and stationary distribution functions, Planet. Space Sci., 30, 433-437.

Tsyganenko, N. A. (1989), A magnetospheric magnetic field model with a warped tail current sheet, Planet. Space. Sci., 37, 5-20, doi:10.1016/0032-0633(89)90066-4.

Walsh, A. P., et al. (2010), Comprehensive ground-based and in situ observations of substorm expansion phase onset, J. Geophys. Res., 115, A00I13, doi:10.1029/2010JA015748.

Walsh, A. P., C. J. Owen, A. N. Fazakerley, C. Forsyth, and I. Dandouras (2011), Average magnetotail electron and proton pitch angle distributions from Cluster PEACE and CIS observations, Geophys. Res. Lett., 38, L06103, doi:10.1029/2011GL046770.

Wang, C.-P., L. R. Lyons, T. Nagai, J. M. Weygand, and R. W. McEntire (2007), Sources, transport, and distributions of plasma sheet ions and 
WALSH ET AL.: PLASMA SHEET ELECTRONS

electrons and dependences on interplanetary parameters under northward interplanetary magnetic field, J. Geophys. Res., 112, A10224, doi:10.1029/2007JA012522.

Wang, C.-P., S. G. Zaharia, L. R. Lyons, and V. Angelopoulos (2013), Spatial distributions of ion pitch angle anisotropy in the near-Earth magnetosphere and tail plasma sheet, J. Geophys. Res. Space Physics, 118 , 244-255, doi:10.1029/2012JA018275.
Wing, S., J. R. Johnson, P. T. Newell, and C.-I. Meng (2005), Dawndusk asymmetries, ion spectra, and sources in the northward interplanetary magnetic field plasma sheet, J. Geophys. Res., 110, A08205, doi:10.1029/2005JA011086.

Wright, A. N., C. J. Owen, C. C. Chaston, and M. W. Dunlop (2008), Downward current electron beam observed by Cluster and FAST, J. Geophys. Res., 113, A06202, doi:10.1029/2007JA012643. 